PROCEEDINGS OF THE

AMERICAN MATHEMATICAL SOCIETY

Volume 126, Number 8, August 1998, Pages 2249-2265

S 0002-9939(98)04710-8

\title{
ON THE NUMBER OF INVARIANT LINES FOR POLYNOMIAL SYSTEMS
}

\author{
ZHANG XIANG AND YE YANQIAN \\ (Communicated by Hal L. Smith)
}

\begin{abstract}
In this paper we will revise the mistakes in a previous paper of Zhang Xikang (Number of integral lines of polynomial systems of degree three and four, J. Nanjing Univ. Math. Biquarterly, Supplement, 1993, pp. 209212 ) for the proof of the conjecture on the maximum number of invariant straight lines of cubic and quartic polynomial differential systems; and also prove the conjecture in a previous paper of the second author (Qualitative theory of polynomial differential systems, Shanghai Science-Technical Publishers, Shanghai, 1995, p. 474) for a certain special case of the $n$ degree polynomial systems. Furthermore, we will prove that cubic and quartic differential systems have invariant straight lines along at most six and nine different directions, respectively, and also show that the maximum number of the directions can be obtained.
\end{abstract}

\section{INTRODUCTION}

In this paper we will consider polynomial systems of degree $n$ :

$$
\dot{x}=P_{n}(x, y), \quad \dot{y}=Q_{n}(x, y),
$$

where $P_{n}$ and $Q_{n}$ are polynomials of degree $n$. The number of invariant algebraic curves of polynomial differential systems (1) is related to its integrability as well as the existence and number of limit cycles (LC). For example, it is well-known that if a quadratic system has two invariant straight lines, it has no LC; if a quadratic system has one invariant straight line, it has at most one LC (see [3]); Kooij [4], [5] has investigated the relationship between invariant straight lines and integrability, and also the existence of LC for cubic systems. Darboux [6] and Jouanolou [7] obtained that for polynomial systems of degree $n$, and for $q$ the number of its independent algebraic invariant straights, when $q>\frac{n(n+1)}{2}\left(\right.$ or $\left.q \geq \frac{n(n+1)}{2}+2\right)$, the system has a first invariant straight (or rational invariant straight). Suo and Sun [8] proved that when (1) has $q=\frac{n(n+1)}{2}-1$ invariant straight lines, it has no LC. Notice that the number of invariant straight lines given in [8] is $O\left(n^{2}\right)$ when $n$ is large. Ye [1] proposed the following:

Received by the editors October 30, 1996.

1991 Mathematics Subject Classification. Primary 34C05.

Key words and phrases. Polynomial differential system, invariant line.

The authors were supported by the National Natural Foundation of the People's Republic of China.

(C)1998 American Mathematical Society 
Conjecture. When $n$ is odd, the system (1) has at most $M_{n}=2 n+2$ invariant straight lines; when $n$ is even, the system (1) has at most $M_{n}=2 n+1$ invariant straight lines.

X. K. Zhang [2] tried to prove this conjecture for $n=3$, 4. His proof depends on the following preliminary property of polynomial systems:

Polynomial systems of degree $n$ have invariant lines along at most $n+1$ different directions.

Dai Guoren gave a counter-example:

$$
\left\{\begin{array}{l}
\dot{x}=x\left(\frac{3}{8} x^{2}+3 x y+y^{2}+2 x+4 y+2\right), \\
\dot{y}=y\left(\frac{3}{8} x^{2}+3 x y+y^{2}+3 x+3 y+2\right),
\end{array}\right.
$$

which has invariant straight lines: $x=0, y=0, y=x, y=-\frac{1}{4} x-1, y=-\frac{3}{4} x-1$, $y=-\frac{3}{2} x-2$, showing that this property does not hold. Hence, even when $n=3,4$, this conjecture is still an open question. The present paper will solve the problem for $n=3,4$ and a special case for $n>4$.

\section{Cubic Differential Systems}

We consider the following cubic differential system:

$$
\begin{cases}\dot{x}=a_{0}+a_{10} x & +a_{01} y+a_{20} x^{2}+a_{11} x y+a_{02} y^{2} \\ & +a_{30} x^{3}+a_{21} x^{2} y+a_{12} x y^{2}+a_{03} y^{3} \triangleq P_{3}(x, y), \\ \dot{y}=b_{0}+b_{10} x & +b_{01} y+b_{20} x^{2}+b_{11} x y+b_{02} y^{2} \\ & +b_{30} x^{3}+b_{21} x^{2} y+b_{12} x y^{2}+b_{03} y^{3} \triangleq Q_{3}(x, y) .\end{cases}
$$

The line $x=c$ is a vertical invariant straight line of (2) if and only if $c$ and the coefficients of (2) satify

$$
\left\{\begin{array}{l}
a_{0}+a_{10} c+a_{20} c^{2}+a_{30} c^{3}=0, \quad a_{01}+a_{11} c+a_{21} c^{2}=0, \\
a_{02}+a_{12} c=0, \quad a_{03}=0 .
\end{array}\right.
$$

The line $y=k x+b$ is an invariant straight line of (2) if and only if $b$ and $k$ satisfy

$$
\left\{\begin{array}{c}
b_{0}+b_{01} b+b_{02} b^{2}+b_{03} b^{3}-k\left(a_{0}+a_{01} b+a_{02} b^{2}+a_{03} b^{3}\right)=0 \\
b_{10}+b_{11} b+b_{12} b^{2}+\left(b_{01}+2 b_{02} b+3 b_{03} b^{2}\right. \\
\left.\quad-a_{10}-a_{11} b-a_{12} b^{2}\right) k-\left(a_{01}+2 a_{02} b+3 a_{03} b^{2}\right) k^{2}=0 \\
b_{20}+b_{21} b+\left(b_{11}+2 b_{12} b-a_{20}-a_{21} b\right) k \\
+\left(b_{02}+3 b_{03} b-a_{11}-2 a_{12} b\right) k^{2}-\left(a_{02}+3 a_{03} b\right) k^{3}=0 \\
b_{30}+\left(b_{21}-a_{30}\right) k+\left(b_{12}-a_{21}\right) k^{2}+\left(b_{03}-a_{12}\right) k^{3}-a_{03} k^{4}=0
\end{array}\right.
$$

If the system (2) has invariant straight lines, by rotating and moving the coordinate systems, we can change any given invariant straight line into $x=0$, which is still an invariant straight line. So, without loss of generality, we always assume $x=0$ is an invariant straight line. Then from (3) we have

$$
a_{0}=a_{01}=a_{02}=a_{03}=0,
$$

hence, formulas (3) and (4) can be rewritten now into

$$
\left(a_{10}+a_{20} c+a_{30} c^{2}\right) c=0, \quad\left(a_{11}+a_{21} c\right) c=0, \quad a_{12} c=0,
$$


$(7)$

$$
\left\{\begin{array}{l}
b_{0}+b_{01} b+b_{02} b^{2}+b_{03} b^{3}=0 \\
b_{10}+b_{11} b+b_{12} b^{2}+\left[b_{01}-a_{10}+\left(2 b_{02}-a_{11}\right) b+\left(3 b_{03}-a_{12}\right) b^{2}\right] k=0 \\
b_{20}+b_{21} b+\left[b_{11}-a_{20}+\left(2 b_{12}-a_{21}\right) b\right] k+\left[b_{02}-a_{11}+\left(3 b_{03}-2 a_{12}\right) b\right] k^{2}=0 \\
b_{30}+\left(b_{21}-a_{30}\right) k+\left(b_{12}-a_{21}\right) k^{2}+\left(b_{03}-a_{12}\right) k^{3}=0
\end{array}\right.
$$

respectively. For convenience, we distinguish the following two cases:

(I) $b_{30}=0, b_{21}=a_{30}, b_{12}=a_{21}$ and $b_{03}=a_{12}$ cannot be simultaneously satisfied;

(II) $b_{30}=0, b_{21}=a_{30}, b_{12}=a_{21}$ and $b_{03}=a_{12}$.

Under condition (I), the fourth equation in (7) has at most three solutions, hence, system (2) has invariant straight lines along at most four different directions.

1. Assume (2) has three vertical invariant straight lines; then we must have (5) and (6) which is reduced to $a_{11}=a_{12}=a_{21}=0 \neq a_{30}$. Then (7) is equivalent to

$$
\left\{\begin{array}{l}
b_{0}+b_{01} b+b_{02} b^{2}+b_{03} b^{3}=0 \\
b_{10}+\left(b_{01}-a_{10}\right) k+\left(b_{11}+2 b_{02} k\right) b+\left(b_{12}+3 b_{03} k\right) b^{2}=0 \\
b_{20}+\left(b_{11}-a_{20}\right) k+b_{02} k^{2}+\left(b_{21}+2 b_{12} k+3 b_{03} k^{2}\right) b=0
\end{array}\right.
$$

and

$$
b_{30}+\left(b_{21}-a_{30}\right) k+b_{12} k^{2}+b_{03} k^{3}=0 .
$$

If for every solution $k_{i}$ of $(9), b_{21}+2 b_{12} k+3 b_{03} k^{2} \neq 0$, for $k=k_{i}$, then corresponding to $k_{i}$, equation (8) has at most one solution $b_{i}$. Hence, system (2) has at most six invariant straight lines along at most four different directions; the distribution of these invariant straight lines is $(3,1,1,1)$.

If there exists a solution $k_{1}$ of $(9)$ such that

$$
b_{20}+\left(b_{11}-a_{20}\right) k+b_{02} k^{2}=0, \quad b_{21}+2 b_{12} k+3 b_{03} k^{2}=0,
$$

but $b_{10}+\left(b_{01}-a_{10}\right) k=0, b_{11}+2 b_{02} k=0$, and $b_{12}+3 b_{03} k=0$ cannot be simultaneously satisfied, then (8) has at most two solutions $b_{1}$ and $b_{2}$ corresponding to $k_{1}$. Furthermore, if there is no other solution of (9) such that (10) is satisfied, the distribution of invariant straight lines of $(2)$ is at most $(3,2,1,1)$.

If there exists a solution $k_{1}$ of (9) such that (10) and

$$
b_{10}+\left(b_{01}-a_{10}\right) k=0, \quad b_{11}+2 b_{02} k=0, \quad b_{12}+3 b_{03} k=0
$$

are simultaneously satisfied by $k=k_{1}$, that is, $k_{1}$ is a root of multiplicity 2 of $b_{21}+2 b_{12} k+3 b_{03} k^{2}=0$, then (8) has at most three solutions corresponding to $k_{1}$, and for any other solution $k_{i}$ of $(9), b_{21}+2 b_{12} k_{i}+3 b_{03} k_{i}^{2} \neq 0$. Hence, the distribution of invariant straight lines of $(2)$ is at most $(3,3,1,1)$, and the cubic system realizing this distribution is

$$
\dot{x}=x(x+1)(x-1), \quad \dot{y}=y(y+1)(y-1),
$$

which has invariant straight lines: $x=0, x=-1, x=1, y=0, y=-1, y=1$, $y=x$ and $y=-x$.

If there exist solutions $k_{1}$ and $k_{2}$ of (9) such that (10) is satisfied by $k=k_{i}(i=$ $1,2)$, then the last equation of (11) is unsatisfied, so (2) has at most two invariant straight lines along the directions $k_{1}$ and $k_{2}$, respectively. If (9) has a third solution $k_{3}$, then $b_{21}+2 b_{12} k_{3}+3 b_{03} k_{3}^{2} \neq 0$. Therefore, the distribution of invariant straight lines of $(2)$ is at most $(3,2,2,1)$, and the cubic system realizing this distribution is

$$
\dot{x}=-x(x-1)(x-2), \quad \dot{y}=y(y-1)(-3 x+2 y+2),
$$


which has invariant straight lines: $x=0, x=1, x=2, y=0, y=1, y=x$, $y=x-1$ and $y=\frac{1}{2} x$.

2. Assume (2) has two vertical invariant straight lines, which reduces conditions (6) to

(i) $a_{12}=0, a_{11} \neq 0, a_{21} \neq 0$.

(ii) $a_{10}=a_{11}=a_{12}=a_{21}=0, a_{30} \neq 0$, or $a_{11}=a_{12}=a_{21}=a_{30}=0, a_{10} \neq 0$.

If condition (ii) is satisfied, similar to the above analysis, when system (2) has invariant straight lines along four directions, the distribution of invariant straight lines of $(2)$ is at most $(2,1,1,1),(2,2,1,1),(2,3,1,1)$ and $(2,2,2,1)$. If condition (i) is satisfied, from (6) and (7) we have

$$
a_{10} a_{21}^{2}-a_{20} a_{11} a_{21}+a_{30} a_{11}^{2}=0
$$

$$
\left\{\begin{array}{l}
b_{0}+b_{01} b+b_{02} b^{2}+b_{03} b^{3}=0 \\
b_{10}+\left(b_{01}-a_{10}\right) k+\left[b_{11}+\left(2 b_{02}-a_{11}\right) k\right] b+\left(b_{12}+3 b_{03} k\right) b^{2}=0 \\
b_{20}+\left(b_{11}-a_{20}\right) k+\left(b_{02}-a_{11}\right) k^{2}+\left[b_{21}+\left(2 b_{12}-a_{21}\right) k+3 b_{03} k^{2}\right] b=0 \\
b_{30}+\left(b_{21}-a_{30}\right) k+\left(b_{12}-a_{21}\right) k^{2}+b_{03} k^{3}=0
\end{array}\right.
$$

Similar to the above analysis, we can obtain that if (2) has invariant straight lines along four directions, the distribution of invariant straight lines of $(2)$ is at most $(2,1,1,1),(2,2,1,1),(2,3,1,1)$, and $(2,3,2,1)$.

3 . Assume (2) has a unique vertical invariant straight line. Then we will get either another direction in which system (2) has at least 2 invariant straight lines (and this case has already been considered), or we may get the case $(1,1,1,1)$.

From the above analysis, we obtain the following

Theorem 1. Under condition (I), system (2) has at most eight invariant straight lines along at most four directions. If (2) has eight invariant straight lines, its distribution is $(3,3,1,1)$ or $(3,2,2,1)$.

Under condition (II), the fourth equation of (7) does not exist. Now, for every solution $b$ of the first equation in (7), we will consider the number of solutions $k$ of (7), and give the maximum number of directions for (2) to have invariant straight lines. Now, (7) is equivalent to

$$
b_{0}+b_{01} b+b_{02} b^{2}+b_{03} b^{3}=0
$$

and

$$
\left\{\begin{array}{l}
b_{10}+b_{11} b+b_{12} b^{2}+\left[b_{01}-a_{10}+\left(2 b_{02}-a_{11}\right) b+2 b_{03} b^{2}\right] k=0 \\
b_{20}+b_{21} b+\left(b_{11}-a_{20}+b_{12} b\right) k+\left(b_{02}-a_{11}+b_{03} b\right) k^{2}=0
\end{array}\right.
$$

If (14) has no solution, system (2) has no invariant straight line in any direction except vertical. Hence, we always assume (14) has at least one solution, and the coefficients of $b$ in the first equation of (15) are not all equal to zero (otherwise, equations (14) and (15) have at most four pairs of solutions, that is, (2) has at most seven invariant straight lines along at most five different directions). If there exists a solution $b$ of (14) such that the coefficients of $k^{i}$ in (15) are all equal to zero, system (2) has infinite invariant straight lines. So, we may suppose that for every solution $b$ of (14), the coefficients of $k^{i}$ in (15) are not all zero. 
(i) For every solution $b_{i}$ of (14), if

$$
b_{01}-a_{10}+\left(2 b_{02}-a_{11}\right) b+2 b_{03} b^{2} \neq 0,
$$

similar to the analysis under the condition (I), system (2) has at most six invariant straight lines along at most four different directions.

(ii) If there exists a solution $b_{1}$ of (14) such that

$$
b_{10}+b_{11} b+b_{12} b^{2}=0, \quad b_{01}-a_{10}+\left(2 b_{02}-a_{11}\right) b+2 b_{03} b^{2}=0,
$$

then (15) has at most two solutions $k_{1}$ and $k_{2}$. For other solutions $b_{i}(i \neq 1)$ of (14), if (16) is satisfied, equation (15) has at most one solution $k$ corresponding to $b_{i}$. Therefore, system (2) has at most seven invariant straight lines along at most five different directions.

(iii) If there exist two solutions $b_{1}, b_{2}$ of (14) such that (17) can be satisfied, equation (15) has at most two solutions corresponding to $b_{1}$ and $b_{2}$, respectively; if (14) has a third solution $b_{3}, b_{3}$ must satisfy (16). Hence, equations (14) and (15) have at most five pairs of solutions $\left(b_{1}, k_{1}\right),\left(b_{1}, k_{2}\right),\left(b_{2}, k_{3}\right),\left(b_{2}, k_{4}\right)$ and $\left(b_{3}, k_{5}\right)$. Thus, system $(2)$ will have at most eight invariant straight lines along at most six different directions (five invariant lines corresponding to the five pairs of solutions plus the three vertical ones). Putting everything together, we can prove the following:

Theorem 2. Under condition (II), system (2) has invariant straight lines along at most six directions. If the maximum number of directions can be obtained, its distribution is $(1,1,1,1,1,1)$.

Proof. From the above discussion, the first conclusion is obvious; we now consider the second conclusion. For the system with three invariant straight lines along a certain direction and one along another, without loss of generality, we may assume it to be

$$
\left\{\begin{array}{l}
\dot{x}=(x+1) x(x-1), \\
\dot{y}=y\left(l x^{2}+m x y+n y^{2}+p x+q y+r\right),
\end{array}\right.
$$

which has invariant straight line $y=k x+b$ with $k$ and $b$ satisfying

$$
\begin{gathered}
k\left(n k^{2}+m k+l-1\right)=0, \\
\left\{\begin{array}{l}
b\left(l+m k+n k^{2}\right)+k(m b+2 n k b+p+q k)=0, \\
b(m b+2 n k b+p+q k)+k\left(n b^{2}+q b+r\right)=-k, \\
b\left(n b^{2}+q b+r\right)=0 .
\end{array}\right.
\end{gathered}
$$

If the coefficients of (19) are not all equal to zero, system (18) has invariant straight lines along at most four directions. Hence, we assume: $n=0, m=0, l=1$; then formulas (19) and (20) are equivalent to

$$
b+k(p+q k)=0, \quad b(p+q k)+k(q b+r)=-k, \quad b(q b+r)=0 .
$$

Equation $(21)$ has at most two solutions $k(k \neq 0)$, that is, system $(18)$ has invariant straight lines along at most four directions.

For the system with two vertical and one horizontal invariant straight lines:

$$
\left\{\begin{array}{l}
\dot{x}=x(x-1)(d x+e y+f), \\
\dot{y}=y\left(l x^{2}+m x y+n y^{2}+p x+q y+r\right),
\end{array}\right.
$$


which has invariant straight lines $y=k x+b$ with $k$ and $b$ satisfying

$$
\left\{\begin{array}{l}
l+m k+n k^{2}=d+e k, \\
b\left(l+m k+n k^{2}\right)+k(m b+2 n k b+p+q k)=-k(d+e k)+k(e b+f), \\
b(m b+2 n k b+p+q k)+k\left(n b^{2}+q b+r\right)=-k(e b+f), \\
b\left(n b^{2}+q b+r\right)=0 .
\end{array}\right.
$$

Similar to the above discussion, we may assume: $n=0, m=e, l=d$; then (23) is equivalent to

$$
\left\{\begin{array}{l}
b(l+m k)+k(m b+p+q k)=-k(l+m k)+k(m b+f), \\
b(m b+p+q k)+k(q b+r)=-k(m b+f), \\
b(q b+r)=0 .
\end{array}\right.
$$

When $b=0$, we have

$$
k(p+q k+l+m k-f)=0, \quad k(r+f)=0 .
$$

When $q b+r=0$, we have

$$
(k+b)(m b+p+l+q k+m k)=0 .
$$

Hence, equation (24) has at most three solutions $k_{i}$ which are not equal to zero, that is, system (22) has invariant straight lines along at most five directions. Therefore, if (2) has invariant straight lines along six directions, it must have a unique invariant straight line along every direction. The cubic system realizing the $(1,1,1,1,1,1)$ distribution can be

$$
\left\{\begin{array}{l}
\dot{x}=x\left(x^{2}+2 y^{2}-1\right), \\
\dot{y}=y\left(x^{2}+2 y^{2}-3 y+1\right),
\end{array}\right.
$$

with oblique invariant straight lines: $y= \pm x+1, y= \pm \frac{1}{2} x+\frac{1}{2}$. This completes the proof of Theorem 2 .

It seems that in a similar way we can get the case $(2,2,2,2)$, but this is impossible, because this will contradict the following property [1] of polynomial systems of degree $n$ :

The number of intersection points of every finite nonsingular invariant line and other invariant lines is at most $n$.

\section{Quartic Differential Systems}

We now consider the following quartic systems:

$$
\left\{\begin{array}{l}
\dot{x}=P_{3}(x, y)+a_{40} x^{4}+a_{31} x^{3} y+a_{22} x^{2} y^{2}+a_{13} x y^{3}+a_{04} y^{4} \triangleq P_{4}(x, y) \\
\dot{y}=Q_{3}(x, y)+b_{40} x^{4}+b_{31} x^{3} y+b_{22} x^{2} y^{2}+b_{13} x y^{3}+b_{04} y^{4} \triangleq Q_{4}(x, y) .
\end{array}\right.
$$

System (25) has a vertical invariant straight line $x=0$ if and only if $a_{0}=a_{01}=$ $a_{02}=a_{03}=a_{04}=0$, and if $x=c(c \neq 0)$ is also a vertical invariant straight line, $c$ must satisfy

$$
\left\{\begin{array}{l}
a_{10}+a_{20} c+a_{30} c^{2}+a_{40} c^{3}=0, \quad a_{11}+a_{21} c+a_{31} c^{2}=0, \\
a_{12}+a_{22} c=0, \quad a_{13}=0 .
\end{array}\right.
$$

System (25) has invariant straight line $y=k x+b$ if and only if $k$ and $b$ satisfy

$$
b_{0}+b_{01} b+b_{02} b^{2}+b_{03} b^{3}+b_{04} b^{4}=0,
$$


(28)

$$
\left\{\begin{array}{c}
b_{10}+b_{11} b+b_{12} b^{2}+b_{13} b^{3}+\left[b_{01}-a_{10}+\left(2 b_{02}-a_{11}\right) b\right. \\
\left.+\left(3 b_{03}-a_{12}\right) b^{2}+\left(4 b_{04}-a_{13}\right) b^{3}\right] k=0 \\
b_{20}+b_{21} b+b_{22} b^{2}+\left[b_{11}-a_{20}+\left(2 b_{12}-a_{21}\right) b+\left(3 b_{13}-a_{22}\right) b^{2}\right] k \\
+\left[b_{02}-a_{11}+\left(3 b_{03}-2 a_{12}\right) b+\left(6 b_{04}-3 a_{13}\right) b^{2}\right] k^{2}=0 \\
b_{30}+b_{31} b+\left[b_{21}-a_{30}+\left(2 b_{22}-a_{31}\right) b\right] k+\left[b_{12}-a_{21}+\left(3 b_{13}-2 a_{22}\right) b\right] k^{2} \\
+\left[b_{03}-a_{12}+\left(4 b_{04}-3 a_{13}\right) b\right] k^{3}=0 \\
b_{40}+\left(b_{31}-a_{40}\right) k+\left(b_{22}-a_{31}\right) k^{2}+\left(b_{13}-a_{22}\right) k^{3}+\left(b_{04}-a_{13}\right) k^{4}=0
\end{array}\right.
$$

Similar to the analysis of the cubic systems, let us first assume equation (29) is not identically zero. Assume system (25) has a maximum number, i.e., four, of invariant straight lines in the vertical direction; we know from (26) that $a_{11}=a_{12}=$ $a_{13}=a_{21}=a_{22}=a_{31}=0, a_{40} \neq 0$. Equation (26) is equivalent to

$$
\left(a_{10}+a_{20} c+a_{30} c^{2}+a_{40} c^{3}\right) c=0 .
$$

Equations (28) and (29) are equivalent to

$$
\begin{gathered}
\left\{\begin{array}{c}
b_{10}+\left(b_{01}-a_{10}\right) k+\left(b_{11}+2 b_{02} k\right) b+\left(b_{12}+3 b_{03} k\right) b^{2}+\left(b_{13}+4 b_{04} k\right) b^{3}=0 \\
b_{20}+\left(b_{11}-a_{20}\right) k \\
+b_{02} k^{2}+\left(b_{21}+2 b_{12} k+3 b_{03} k^{2}\right) b \\
+\left(b_{22}+3 b_{13} k+6 b_{04} k^{2}\right) b^{2}=0 \\
b_{30}+\left(b_{21}-a_{30}\right) k+b_{12} k^{2}+b_{03} k^{3}+\left(b_{31}+2 b_{22} k+3 b_{13} k^{2}+4 b_{04} k^{3}\right) b=0
\end{array}\right. \\
b_{40}+\left(b_{31}-a_{40}\right) k+b_{22} k^{2}+b_{13} k^{3}+b_{04} k^{4}=0
\end{gathered}
$$

Using the same procedure as in the case $n=3$, we can similarly prove that system (25) has at most nine invariant straight lines (but the computation is more complicated; due to limited space, the details are omitted), their distributions may be $(4,1,1,1,1),(4,2,2,1),(4,2,1,1,1),(4,3,2),(4,3,1,1),(4,4)$ and $(4,4,1)$, and the realizing systems are respectively

$$
\left\{\begin{array}{l}
\dot{x}=(x+1) x(x-1)(x-2), \\
\dot{y}=y\left(x^{3}-x y-2 y^{2}+x+6 y-4\right),
\end{array}\right.
$$

which has oblique invariant straight lines: $y=x+1, y=-x+1, y=-\frac{1}{2} x+1$; and

$$
\left\{\begin{array}{l}
\dot{x}=(x+1) x(x-1)(x-2) \\
\dot{y}=y(y-1)\left(4 x^{2}+4 x y-16 y^{2}-2 x+8 y\right)
\end{array}\right.
$$

which has oblique invariant straight lines $y=\frac{1}{2} x+\frac{1}{2}, y=\frac{1}{2} x, y=-\frac{1}{2} x+\frac{1}{2}$; and

$$
\left\{\begin{array}{l}
\dot{x}=(x+1) x(x-1)(x-2), \\
\dot{y}=y(y-1)\left(2 x^{2}+x y-2 y^{2}+x-2 y-2\right),
\end{array}\right.
$$

which has oblique invariant straight lines: $y=\frac{1}{2} x, y=x, y=-x$; and

$$
\dot{x}=(x+1) x(x-1)(x-2), \quad \dot{y}=(y+1) y(y-1)(4 x-3 y-2),
$$

which has oblique invariant lines $y=x, y=x-1$; and

$$
\dot{x}=(x+1) x(x-1)(x+2), \quad \dot{y}=(y+1) y(y-1)(x-1),
$$


which has oblique invariant lines $y=-x-1, y=x+1$ (note that the system realizing $(4,3,1,1)$ distribution must have a finite singular invariant line); and

$$
\dot{x}=(x-2)(x-1)(x+1)(x+2), \quad \dot{y}=(y-2)(y-1)(y+1)(y+5),
$$

which has no oblique line; and

$$
\dot{x}=(x-2)(x-1)(x+1)(x+2), \quad \dot{y}=(y-2)(y-1)(y+1)(y+2),
$$

which has a unique oblique line $y=x$.

As for the quartic system having at most three vertical invariant straight lines, we may get either another direction in which we have 4 invariant straight lines (and this case has already been considered), or the number of invariant straight lines along the other direction (excepting vertical) is less than or equal to 3 . We may have $(3,3,3),(3,3,2,1)$, or $(3,3,1,1,1)$ distributions which can be realized by

$$
\left\{\begin{array}{l}
\dot{x}=(x+1) x(x-1)(x-2 y), \\
\dot{y}=(y+1) y(y-1)(-2 x+y),
\end{array}\right.
$$

which has oblique invariant straight lines: $y=x, y=x \pm 1$; and the system

$$
\left\{\begin{array}{l}
\dot{x}=(x+1) x(x-1)(y+1), \\
\dot{y}=(y+1) y(y-1)(3 x-2 y+1),
\end{array}\right.
$$

with oblique invariant straight lines: $y=x, y=x+1, y=\frac{1}{2} x+\frac{1}{2}$; and the system

$$
\left\{\begin{array}{l}
\dot{x}=(x+1) x(x-1)(x-2 y+1), \\
\dot{y}=(y+1) y(y-1)(x-2 y+1),
\end{array}\right.
$$

with oblique invariant straight lines: $y= \pm x, y=\frac{1}{2} x+\frac{1}{2}$ which is a singular one. Similar to the end of $\S 2$, we see that $(2,2,2,2,2),(3,3,3,1)$ and $(3,3,2,2)$ are impossible.

Next, let us assume equation (29) is identically zero, that is, $b_{40}=0, b_{31}=a_{40}$, $b_{22}=a_{31}, b_{13}=a_{22}$, and $b_{04}=a_{13}$. Similar to the case of $n=3$, we distinguish several different cases.

For the systems with four vertical and three horizontal invariant straight lines, by parallel translation and stretching of coordinate systems, we may assume

$$
\dot{x}=(x+1) x\left(x-c_{1}\right)\left(x-c_{2}\right), \quad \dot{y}=(y+1) y\left(y-b_{1}\right)(l x+m y+n),
$$

where $c_{1}, c_{2}, b_{1}>0$.

If system (32) has oblique invariant line, it must pass through three intersection points of horizontal and vertical invariant lines. When $c_{1}=1, c_{2}=2$ and $b_{1}=1$ cannot be simultaneously satisfied, system (32) has at most nine invariant straight lines; their locus can be shown in Figure 1, in which we have at most two oblique invariant straight lines, and they are not parallel. Otherwise, an oblique invariant line can be found on which there are five intersection points with other invariant lines.

Remark. For the system

$$
\dot{x}=(x+1) x(x-1)(x-c), \quad \dot{y}=(y+1) y(y-1)(x-c),
$$

where $c \neq-1,0,1$, the number of intersection points of the singular invariant line $x=c$ with invariant lines $y=0, y= \pm 1, y= \pm x$ is five. 

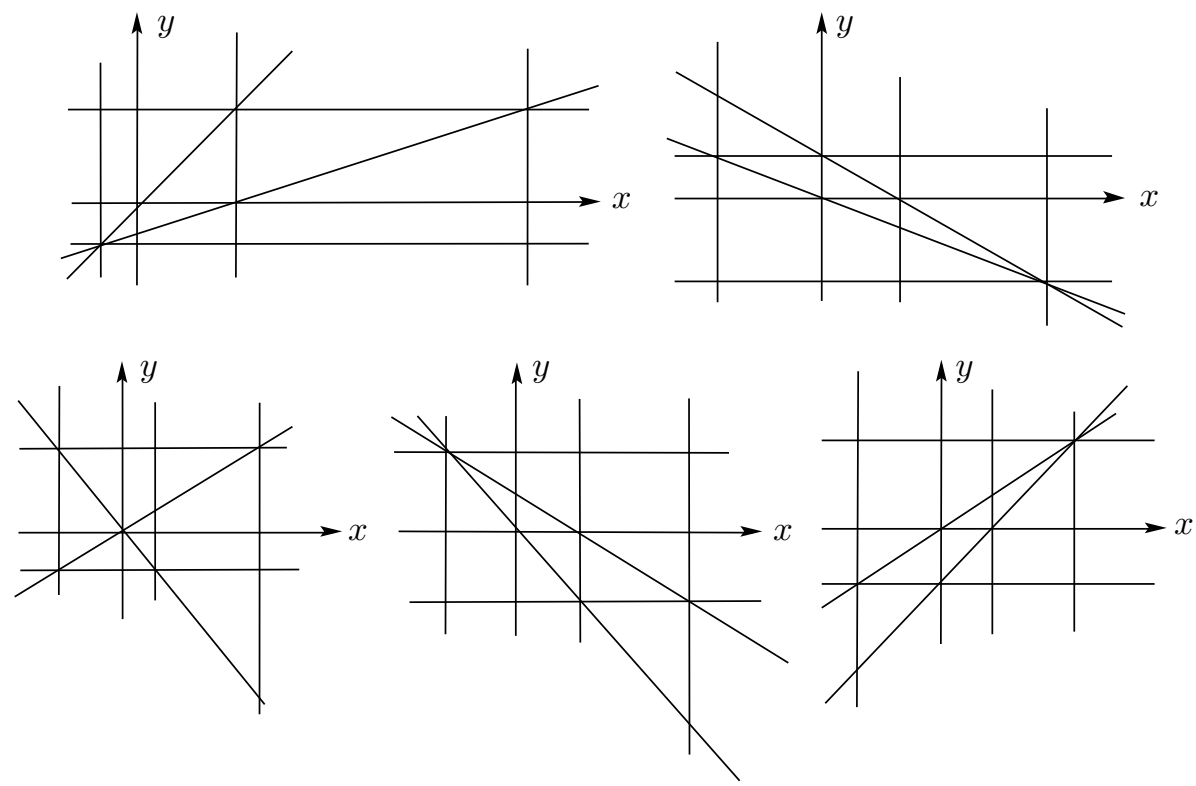

FiguRE 1

Now, we study the system (32) with $c_{1}=1, c_{2}=2$, and $b_{1}=1$. Assume $y=k x+b$ is an invariant straight line of (32) along a direction other than vertical and horizontal. Then $k, b, l, m$ and $n$ must satisfy

$$
\left\{\begin{array}{l}
k^{2}(l+m k)=1, \\
k(2 m b k+n k+b l)+2 b k(l+m k)=-2, \\
k b(m b+n)+2 b(2 m b k+n k+b l)+\left(b^{2}-1\right)(l+m k)=-1, \\
2 b^{2} k(m b+n)+\left(b^{2}-1\right)(2 m b k+n k+b l)=2 k,
\end{array}\right.
$$

and

$$
b\left(b^{2}-1\right)(m b+n)=0 .
$$

If $m b+n=0,(33)$ becomes

$$
\left\{\begin{array}{l}
k^{2}(l+m k)=1, \quad 3 b k(l+m k)=-2, \\
\left(3 b^{2}-1\right)(l+m k)=-1, \quad b\left(b^{2}-1\right)(l+m k)=2 k,
\end{array}\right.
$$

which has no solution as is easily seen, that is, under the condition $m b+n=0$, system (32) has at most seven invariant straight lines.

From the equations (33) and (34), we obtain
(a) $b=0, k= \pm 1, l \pm m=1, n=-2$
(b) $b=1, k=-1, l-m=1, n+m=1$;
(c) $b=-1, k=1, l+m=1, n-m=1$.

Obviously, any three conditions in (a)-(c) cannot be simultaneously satisfied. Thus, equations (33) and (34) have at most two pairs of solutions, that is, system (32) has at most 2 oblique lines. Moreover, system (32) has at most nine invariant straight lines. 
Next, we may assume the system with four vertical and two horizontal invariant straight lines to be:

$$
\left\{\begin{array}{l}
\dot{x}=(x+1) x(x-1)(x-2) \\
\dot{y}=y(y-1)\left(l x^{2}+m x y+n y^{2}+p x+q y+r\right) .
\end{array}\right.
$$

If $y=k x+b$ is an invariant straight line of (35), $k$ and $b$ must satisfy

$$
\left\{\begin{array}{l}
k\left(l+m k+n k^{2}\right)=1, \\
k(m b+2 n k b+p+q k)+(2 b-1)\left(l+m k+n k^{2}\right)=-2, \\
k^{2}\left(n b^{2}+q b+r\right)+(2 b-1) k(m b+2 n k b+p+q k) \\
\quad+b(b-1)\left(l+m k+n k^{2}\right)=-k, \\
(2 b-1) k\left(n b^{2}+q b+r\right)+b(b-1)(m b+2 n k b+p+q k)=2 k,
\end{array}\right.
$$

and

$$
b(b-1)\left(n b^{2}+q b+r\right)=0 .
$$

If $b=0$, solving (36) gives $r=-2, k=\frac{1}{2}, \pm 1$, the system (35) has nine invariant straight lines. If $b=1$, solving (36) gives $n+q+r=2, k=-\frac{1}{2}$, \pm 1 , system (35) has also nine invariant lines.

If $n b^{2}+q b+r=0$, equation (36) becomes

$$
\left\{\begin{array}{l}
k\left(l+m k+n k^{2}\right)=1, \\
k(m b+2 n k b+p+q k)+(2 b-1)\left(l+m k+n k^{2}\right)=-2, \\
(2 b-1) k(m b+2 n k b+p+q k)+b(b-1)\left(l+m k+n k^{2}\right)=-k, \\
b(b-1)(m b+2 n k b+p+q k)=2 k .
\end{array}\right.
$$

From the above equations, we obtain

$$
(2 b-1)(1-2 k-2 b)+b(b-1)=-k^{2}, \quad b(b-1)(1-2 k-2 b)=2 k^{3},
$$

and solving these equations gives

$$
b=\frac{1}{2}, \quad k= \pm \frac{1}{2}
$$

or

$$
2 k(1-2 k-2 b)=b(b-1) .
$$

When (38) is satisfied, we have

$$
\left\{\begin{array}{l}
l+\frac{1}{2} m+\frac{1}{4} n=2, \quad m+n+2 p+q=-8, \quad \frac{1}{4} n+\frac{1}{2} q+r=0, \\
l-\frac{1}{2} m+\frac{1}{4} n=-2, \quad m-n+2 p-q=8 .
\end{array}\right.
$$

Hence, we know from (40) that $m=4, p=-2, l=-\frac{1}{4} n, q=-n-8, r=\frac{1}{4} n+4$.

Furthermore, we have

$$
n b^{2}-(n+8) b+\frac{1}{4} n+4=0,
$$

which has two roots: $b=\frac{1}{2}, b=\frac{n+16}{2 n}$. When $b=\frac{n+16}{2 n}$, from (39) we have

$$
4 k^{2}+\frac{32}{n} k+\frac{16^{2}-n^{2}}{4 n^{2}}=0,
$$


which has roots $k=-\frac{4}{n} \pm \frac{1}{4}$. Combining the first equation of (37), we obtain the equations

$$
3 n^{2}+32 n-256=0, \quad 3 n^{2}-32 n-256=0,
$$

which have roots $n=\frac{16}{3},-16$, and $n=-\frac{16}{3}, 16$, respectively. When $n=-16$, then $k=\frac{1}{2}, b=0, l=4, q=8, r=0$. Thus equations (36) and (36') have at most three pairs of solutions. Therefore, system (35) has at most three invariant straight lines along directions other than the vertical and horizontal ones. Moreover, the corresponding system has at most 9 invariant lines.

Finally, we may assume the system with four vertical and one horizontal invariant straight lines to be:

$$
\left\{\begin{array}{l}
\dot{x}=(x+1) x(x-1)(x-2), \\
\dot{y}=y\left(d x^{3}+g x^{2} y+f x y^{2}+h y^{3}+l x^{2}+m x y+n y^{2}+p x+q y+r\right),
\end{array}\right.
$$

which has invariant straight line $y=k x+b$ with $k$ and $b$ satisfying

$$
\begin{gathered}
k\left(d f+g k+f k^{2}+h k^{3}\right)=k \\
\left\{\begin{array}{l}
k\left(g b+2 f b k+3 h b k^{2}+l+m k+n k^{2}\right)+b\left(d+g k+f k^{2}+h k^{3}\right)=-2 k \\
k\left(f b^{2}+3 h b^{2} k+m b+2 n b k+p+q k\right) \\
+b\left(g b+2 f b k+3 h b k^{2}+l+m k+n k^{2}\right)=-k \\
k\left(h b^{3}+n b^{2}+q b+r\right)+b\left(f b^{2}+3 h b^{2} k+m b+2 n b k+p+q k\right)=2 k \\
b\left(h b^{3}+n b^{2}+q b+r\right)=0
\end{array}\right.
\end{gathered}
$$

From the above discussion, we only need to investigate the case with one invariant straight line along every other direction excepting vertical, otherwise, system (41) can be changed into previous cases. If the coefficients of (42) are not all equal to zero, system (41) has at most eight invariant straight lines along at most five directions, hence, we may assume $h=g=f=0, d=1$. Then (43) is equivalent to

$$
b=0, \quad l+m k+n k^{2}=-2, \quad p+q k=-1, \quad r=2,
$$

Or

$$
\begin{gathered}
n b^{2}+q b+r=0, \\
\left\{\begin{array}{l}
k\left(l+m k+n k^{2}\right)+b=-2 k, \\
k(m b+2 n b k+p+q k)+b\left(l+m k+n k^{2}\right)=-k, \\
b(m b+2 n b k+p+q k)=2 k .
\end{array}\right.
\end{gathered}
$$

For every solution $b_{i}$ of (45) (which has at most two solutions), solving (46) gives

$$
k=b_{i}, \quad k=-b_{i}, \quad k=-\frac{1}{2} b_{i} .
$$

Correspondingly, aside from (45), $b_{i}$ must satify

$$
l+m b_{i}+n b_{i}^{2}=-3, \quad m b_{i}+2 n b_{i}^{2}+p+q b_{i}=2,
$$

or

$$
l-m b_{i}+n b_{i}^{2}=-1, \quad m b_{i}-2 n b_{i}^{2}+p-q b_{i}=-2,
$$


or

$$
l-\frac{1}{2} m b_{i}+\frac{1}{4} n b_{i}^{2}=0, \quad m b_{i}-n b_{i}^{2}+p-\frac{1}{2} q b_{i}=-1 .
$$

If (44) has two roots $k$, we must have $p=-1, q=0, r=2$. If (45) and (46) have two different solutions $k$, it is necessary that for the solutions $b_{i}(i=1,2)$ of (45), at least two equations in (47)-(49) are simultaneously satisfied; we assume they are $(47)(i=1)$ and $(49)(i=2)$. Other cases can be similarly discussed, and so omitted. Since (45) gives $n b_{i}^{2}=-2(i=1,2)$, solving $(47)(i=1)$ and $(49)(i=2)$, we obtain $l=-8, m b_{1}=7$, and $l=-\frac{1}{2}, m b_{2}=-2$, respectively, which is a contradiction. Therefore, when (44) has two roots $k$, equations (45) and (46) have only one solution. Similarly, we can prove that when (44) has a unique root, at most two equations in (47)-(49) can be simultaneously satisfied; if three equations in (47)-(49) are simultaneously satisfied, then other equations cannot be satisfied, and (44) has no root. Therefore, for any given set of coefficients, equations (44)-(46) have at most three different solutions $k$, that is, system (41) has invariant straight lines along at most five directions with one line in each direction excepting vertical. Moreover, system (41) has at most eight invariant straight lines.

As for the quartic system having at most three vertical invariant straight lines, and the number of invariant straight lines along the other direction is less than or equal to that along the vertical, it can be similarly proved that the system has at most nine invariant straight lines; the details are omitted.

As for the maximum number of directions for the invariant straight lines of quartic differential systems, we have the following:

Theorem 3. If system (25) has no parallel invariant straight lines, then it has invariant straight lines along at most nine different directions. Moreover, the maximum number of directions can be obtained.

Proof. Without loss of generality, we may assume system (25) has a unique invariant straight line along vertical and horizontal directions, respectively; then $b_{0}=b_{10}=$ $b_{20}=b_{30}=b_{40}=0$ in (27)-(29). Furthermore, we may assume $b_{31}=a_{40}, b_{22}=a_{31}$, $b_{13}=a_{22}$ and $b_{04}=a_{13}$, otherwise, system (25) has invariant straight lines along at most five directions. Formulas (27) and (28) are equivalent to

$$
\left\{\begin{array}{l}
b=0, \quad b_{01}-a_{10}=0, \quad b_{11}-a_{20}+\left(b_{02}-a_{11}\right) k=0, \\
b_{21}-a_{30}+\left(b_{12}-a_{21}\right) k+\left(b_{03}-a_{12}\right) k^{2}=0
\end{array}\right.
$$

or

$$
b_{01}+b_{02} b+b_{03} b^{2}+b_{04} b^{3}=0
$$

$$
\left\{\begin{aligned}
& b_{31} b+\left(b_{21}-a_{30}+b_{22} b\right) k+\left(b_{12}-a_{21}+b_{13} b\right) k^{2}+\left(b_{03}-a_{12}+b_{04} b\right) k^{3}=0 \\
& b_{21} b+b_{22} b^{2}+ {\left[b_{11}-a_{20}+\left(2 b_{12}-a_{21}\right) b+2 b_{13} b^{2}\right] k } \\
&+\left[b_{02}-a_{11}+\left(3 b_{03}-2 a_{12}\right) b+3 b_{04} b^{2}\right] k^{2}=0 \\
& b_{11} b+b_{12} b^{2}+ b_{13} b^{3}+\left[-a_{10}+\left(b_{02}-a_{11}\right) b+\left(2 b_{03}-a_{12}\right) b^{2}+2 b_{04} b^{3}\right] k=0
\end{aligned}\right.
$$

We may assume $b_{01} \neq 0$, otherwise, the situation will be very simple. For the solution $b_{1}$ of (51), if (52) has three solutions $k$, it is necessary that $b_{21}+b_{22} b_{1}=0$, 
and

$$
\left\{\begin{array}{l}
b_{02}-a_{11}+\left(3 b_{03}-2 a_{12}\right) b_{1}+3 b_{04} b_{1}^{2}=0, \\
-b_{01}+\left(b_{02}-a_{11}\right) b_{1}+\left(2 b_{03}-a_{12}\right) b_{1}^{2}+2 b_{04} b_{1}^{3}=0 .
\end{array}\right.
$$

For another solution $b_{2}\left(\neq b_{1}\right)$ of $(51)$, since $b_{21}+b_{22} b_{2} \neq 0$ and equation (52) has at most two solutions $k$, it is necessary that

$$
-b_{01}+\left(b_{02}-a_{11}\right) b_{2}+\left(2 b_{03}-a_{12}\right) b_{2}^{2}+2 b_{04} b_{2}^{3}=0 .
$$

From (51)-(54), we obtain

$$
\left\{\begin{array}{l}
b_{1}=-\frac{b_{02}}{a_{12}}, \quad b_{2}=-\frac{a_{11}}{a_{12}}, \quad b_{3}=-\frac{a_{11} b_{02}}{3 a_{12}\left(2 b_{02}-a_{11}\right)}, \\
a_{10}=b_{01}=\frac{a_{11} b_{02}}{3 a_{12}}, \quad b_{03}=\frac{a_{12}}{3}\left(\frac{2 b_{02}}{a_{11}}-\frac{a_{11}}{b_{02}}+2\right), \quad b_{04}=\frac{a_{12}^{2}}{3}\left(\frac{2}{a_{11}}-\frac{1}{b_{02}}\right),
\end{array}\right.
$$

where $a_{12} \neq 0,2 b_{02}-a_{11} \neq 0$ (if $a_{12}=0$, we have $b_{02}+a_{11}=0$, hence $b_{01}=0$, which contradicts the assumption; $2 b_{02}-a_{11}=0$ leads to $b_{04}=0$, it is very simple). Since $b_{11}+b_{12} b+b_{13} b^{2}=0$ has at most two roots, for the third solution of (51), equation (52) has at most one solution $k$. Hence, equations (51) and (52) have at most six pairs of different solutions $\left(b_{i}, k_{i}\right)$. Since $b_{1} \neq b_{2}$, then $b_{02} \neq a_{11}$, equation (50) has at most one pair of solutions $(0, k)$. Therefore, equations (50)-(52) have at most seven different solutions $k$, that is, system (25) has invariant straight lines along at most nine different directions. If (50) has two different solutions, it is necessary that $b_{02}=a_{11}, b_{11}=a_{20}$, and $b_{01}=a_{10}$. We know from the above proof that (52) has at most five different solutions $k$. Hence, system (25) has invariant straight lines along at most nine different directions. The example

$$
\left\{\begin{array}{l}
\dot{x}=x\left(15 x^{3}-99 x^{2} y-135 x y^{2}+99 y^{3}-13 x^{2}+165 x y-108 y^{2}-30 x+66 y-8\right), \\
\dot{y}=y\left(15 x^{3}-99 x^{2} y-135 x y^{2}+99 y^{3}+36 x^{2}+165 x y-157 y^{2}-30 x+66 y-8\right),
\end{array}\right.
$$

with invariant straight lines $x=0, y=0, y= \pm x, y=-\frac{1}{2} x+1, y=3 x+1$, $y=\frac{2}{3} x+\frac{2}{9}, y=-\frac{1}{9} x+\frac{2}{9}, y=\frac{5}{11} x+\frac{4}{11}$ shows that the quartic differential system can have invariant straight lines along nine different directions. This completes the proof of Theorem 3 .

\section{Polynomial differential systems of Degree $n$}

Now, for the general polynomial systems of degree $n$ :

$$
\left\{\begin{array}{l}
\dot{x}=a_{0}+\sum_{l=1}^{n} P_{l}(x, y) \triangleq P(x, y) \\
\dot{y}=b_{0}+\sum_{l=1}^{n} Q_{l}(x, y) \triangleq Q(x, y)
\end{array}\right.
$$

where

$$
P_{l}=\sum_{i+j=l} a_{i j} x^{i} y^{j}, \quad Q_{l}=\sum_{i+j=l} b_{i j} x^{i} y^{j}
$$

$a_{i j}, b_{i j}$ are constants. Under the condition that (56) has maximum number, i.e., $n$, of invariant straight lines along two directions, respectively, we will prove the conjecture of [1]. As for the other cases, the computation will be more complicated. ${ }^{1}$

\footnotetext{
${ }^{1}$ We know quite recently that J. Llibre and others have given examples showing that when $n=5$, the system (56) can have 14 invariant straight lines, so the conjecture in [1] is false for $n=5$.
} 
When (56) has vertical invariant straight line $x=0$, if $x=c$ is an invariant straight line of (56), the coefficients of the systems (56) and $c$ must satisfy $a_{0}=$ $a_{01}=a_{02}=\cdots=a_{0 n}=0$, and

$$
\begin{gathered}
\left(a_{10}+a_{20} c+\cdots+a_{n 0} c^{n-1}\right) c=0 \\
\left\{\begin{array}{c}
\left(a_{11}+a_{21} c+\cdots+a_{(n-2) 1} c^{n-3}+a_{(n-1) 1} c^{n-2}\right) c=0 \\
\left(a_{12}+a_{22} c+\cdots+a_{(n-2) 2} c^{n-3}\right) c=0 \\
\cdots \quad \cdots \quad \cdots \\
\left(a_{1(n-2)}+a_{2(n-2)} c\right) c=0, \quad a_{1(n-1)} c=0 .
\end{array}\right.
\end{gathered}
$$

If (56) has $n$ vertical invariant straight lines, we have

$$
\begin{aligned}
& a_{11}=a_{12}=\cdots=a_{1(n-2)}=a_{1(n-1)}=0, a_{21}=a_{22}=\cdots=a_{2(n-2)}=0 \\
& \cdots, a_{(n-2) 1}=a_{(n-2) 2}=a_{(n-1) 1}=0 . \\
&
\end{aligned}
$$

So, if (56) has invariant straight lines $y=k x+b$ along other directions, $k$ and $b$ must satisfy

$$
b_{0}+b_{01} b+b_{02} b^{2}+\cdots+b_{0(n-1)} b^{n-1}+b_{0 n} b^{n}=0,
$$

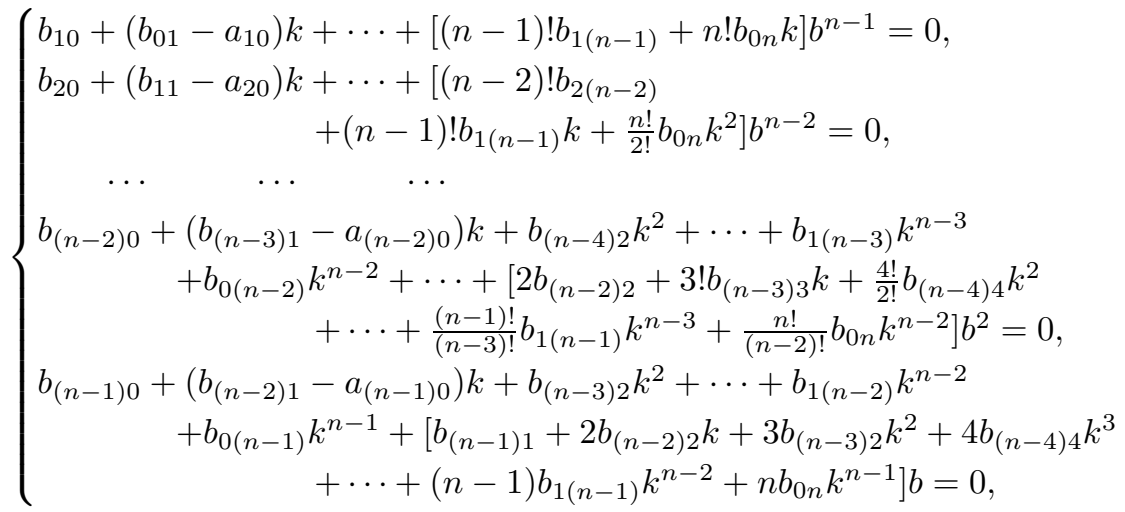

$$
\begin{aligned}
& b_{n 0}+\left(b_{(n-1) 1}-a_{n 0}\right) k+b_{(n-2) 2} k^{2}+b_{(n-3) 3} k^{3}+b_{(n-4) 4} k^{4} \\
& +\cdots+b_{1(n-1)} k^{n-1}+b_{0 n} k^{n}=0 .
\end{aligned}
$$

We assume (59) has a root $k_{1}$ such that (56) has $n$ invariant straight lines along the $k_{1}$ direction, that is, when $k=k_{1}$, the coefficients of (58) are all equal to zero, 
equation (57) has $n$ roots. Therefore, $k_{1}$ satisfies the following equations:

(60)

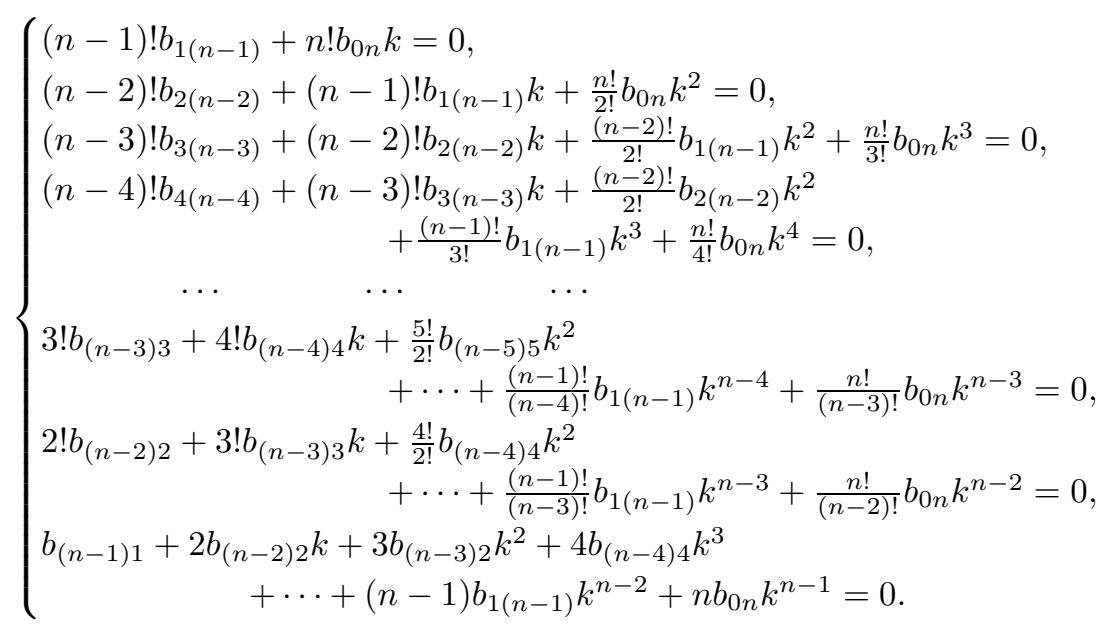

From (60), we know that

$$
\begin{aligned}
k_{1}=-\frac{b_{1(n-1)}}{n b_{0 n}}, \quad b_{k(n-k)} & =\frac{(n-1) !}{k ! n^{k-1}(n-k) !} \frac{b_{1(n-1)}^{k}}{b_{0 n}^{k-1}} \\
& =\frac{C_{n}^{k}}{n^{k}} \frac{b_{1(n-1)}^{k}}{b_{0 n}^{k-1}}, k=2,3, \cdots, n-1 .
\end{aligned}
$$

Substituting (61) into (59), we obtain

$$
\begin{aligned}
b_{n 0} & +a_{n 0} \frac{b_{1(n-1)}}{n b_{0 n}}+\frac{b_{1(n-1)}^{n}}{n^{n} b_{0 n}^{n-1}}\left[-C_{n}^{n-1}+C_{n}^{n-2}-C_{n}^{n-3}+C_{n}^{n-4}+\cdots\right. \\
& \left.+(-1)^{n-4} C_{n}^{4}+(-1)^{n-3} C_{n}^{3}+(-1)^{n-2} C_{n}^{2}+(-1)^{n-1} C_{n}^{1}+(-1)^{n}\right]=0,
\end{aligned}
$$

which is equivalent to

$$
b_{n 0}=\frac{b_{1(n-1)}^{n}}{n^{n} b_{0 n}^{n-1}}-a_{n 0} \frac{b_{1(n-1)}}{n b_{0 n}} .
$$

From (61) and (63), equation (59) can be rewritten as

$$
\left(n b_{0 n} k+b_{1(n-1)}\right)^{n}-n^{n} a_{n 0} b_{0 n}^{n-1} k-n^{n-1} a_{n 0} b_{0 n}^{n-2} b_{1(n-1)}=0,
$$

which can be decomposed into

$$
\left(n b_{0 n} k+b_{1(n-1)}\right)\left[\left(n b_{0 n} k+b_{1(n-1)}\right)^{n-1}-n^{n-1} a_{n 0} b_{0 n}^{n-2}\right]=0 ;
$$

equation (64), i.e., (59), has another root excepting $k_{1}$ if and only if

$$
\left(n b_{0 n} k+b_{1(n-1)}\right)^{n-1}-n^{n-1} a_{n 0} b_{0 n}^{n-2}=0 .
$$

When $n=2 m$, equation (65) has a unique real root

$$
k_{2}=-\frac{b_{1(n-1)}}{n b_{0 n}}+\sqrt[n-1]{\frac{a_{n 0}}{b_{0 n}}} .
$$


Since $k_{1}$ is a root of multiple $n-1$ of the last equation in (60), corresponding to $k_{2}$, equations (57) and (58) have at most one root, that is, except for the vertical and $k_{1}$ directions, there exists at most one direction such that (56) has at most one invariant straight line along this direction. So, when $n=2 m$, system (56) has at most $2 n+1$ invariant straight lines.

When $n=2 m+1$, if $a_{n 0} b_{0 n}^{n-2}<0$, equation (65) has no root and system (56) has at most $2 n$ invariant straight lines. If $a_{n 0}=0$, equation (65) has a unique solution $k_{2}\left(=k_{1}\right)$, that is, $k_{1}$ is a root of multiple $n$ of (59). So, system (56) has at most $2 n$ invariant straight lines (no oblique one). If $a_{n 0} b_{0 n}^{n-2}>0$, equation (65) has only two real solutions

$$
k_{2,3}=-\frac{b_{1(n-1)}}{n b_{0 n}} \pm \sqrt[n-1]{\frac{a_{n 0}}{b_{0 n}}}
$$

Similar to the case for $n=2 m$, except for the vertical and $k_{1}$ directions, there exist at most two directions such that (56) has at most one invariant straight line along each direction. Therefore, when $n=2 m+1$, system (56) has at most $2 n+2$ invariant straight lines.

\section{ADDED IN PROOF}

We learned quite recently that

1. [9] and [10] have also given a geometrical proof of the maximum number of invariant straight lines for quartic systems.

2. [11] has got an interesting relation $\beta(n)=\alpha(n-1)+1$, where $\alpha(n)$ and $\beta(n)$ denote the maximum number and the maximum number of slopes for the invariant straight lines of an $n$-polynomial system, respectively. So from $\alpha(2)=5, \alpha(3)=8$ and $\alpha(4)=9$, we can get easily $\beta(3)=6, \beta(4)=9$ and $\beta(5)=10$.

\section{ACKNowledgement}

The authors would like to thank the referee for many helpful comments.

\section{REFERENCES}

1. Ye Yanqian, Qualitative Theory of Polynomial Differential Systems, Shanghai ScienceTechnical Publisher, Shanghai, 1995.

2. Zhang Xikang, Number of integral lines of polynomial systems of degree three and four, J. Nanjing Univ. Math. Biquarterly, Supplement, 1993, 209-212. MR 95a:34047

3. Ye Yanqian et al., Theory of Limit Cycles, Trans. Math. Monographs, Amer. Math. Soc., 66(1986). MR 88e:58080

4. R.E. Kooij, Some new properties of cubic systems, Delft Univ. Tech., Research Report, preprint, 1991.

5. R. E. Kooij, Real cubic systems with four line invariants, Delft Univ. Tech., Research Report, 91 79, 1991.

6. G. Darboux, Mémoire sur les equations differentielles algebriques du premier order et du premier degré, Bull. des Sc. Math., 1878, pp.60-96; 123-144; 151-200.

7. J. P. Jouanolou, Equations de phaff algebriques, Lect. Notes in Math., 708(1979).

8. Suo Guangjian and Sun Jifang, The n-th degree differential system with $\frac{(n-1)(n+2)}{2}$ straight line solutions has no limit cycles, Proc. of the Conf. on Ordinary Differential Equations and Contral Theory, Wuhan, 1987.

9. J. Sokulski, On the number of invariant lines of polynomial vector fields, Nonlinearity, 9(1996), 479-485. MR 96m:34081 
10. Dai Guoren, Two estimations of the number of invariant straight lines for $\mathrm{n}$-th polynomial differential systems, Acta Mathematica Scientia, 16(1996), 2, 232-240.

11. J. C. Artes and J. Llibre, On the number of slopes of invariant straight lines for polynomial differential systems, J. Nanjing Univ., Math. Biquarterly, 13(1996), 2, 143-149. CMP 97:10

Department of Mathematics, Nanjing Normal University, Nanjing, China 210097

E-mail address: xzhang@pine.njnu.edu.cn

Department of Mathematics, Nanjing University, Nanjing, China 210008 